\title{
Variabilidad anatómica de los senos maxilares y de estructuras involucradas en su vía de drenaje
}

\section{Variability of the maxillary sinuses and structures involved in their drainage path}

\author{
Pinares Toledo J*, Carrillo Porras T*, Guzmán Zuluaga IC**, Ardila Medina CM**, \\ Guzmán Zuluaga $\mathrm{CL}^{*}$
}

\section{RESUMEN}

Introducción: La enfermedad de los senos paranasales, particularmente la de los senos maxilares, es común y puede causar sintomatología que simula patología dental.

Objetivo: Determinar las variaciones anatómicas del seno maxilar y de las estructuras involucradas en su drenaje, utilizando tomografía computarizada de haz de cono (TCHC).

Materiales y Métodos: El estudio incluyó los exámenes descriptivos de TCHC en 48 pacientes. En todos los casos analizados se realizó un estudio sistemático de los senos maxilares y de las estructuras anatómicas involucradas en su vía de drenaje, en los planos coronal, sagital y axial.

Resultados y Conclusiones: Se identificaron variaciones morfológicas en el septo interno del seno maxilar, la celdilla de Haller, el proceso unciforme y la bulla etmoidal. Se observó presencia de septo interno del seno maxilar y de celdillas de Haller en el 76,74\% y 16,28\% de los individuos, respectivamente. El proceso unciforme horizontal se observó en el $79,07 \%$ de los sujetos y el vertical en el $20,93 \%$. Además, se identificaron bullas etmoidales bilaterales en el $62,79 \%$ de los casos. Los senos paranasales presentan variaciones anatómicas marcadas fundamentalmente por su desarrollo prenatal y postnatal, ligados a la neumatización general del neumocráneo y a estructuras que inhiben o permiten su desarrollo. La tomografía computarizada de haz de cono proporciona imágenes que permiten una visualización y evaluación adecuada del seno maxilar y del complejo osteomeatal.

Palabras clave: Seno maxilar, vía de drenaje, variaciones anatómicas, tomografía computarizada de haz de cono.

\section{SUMMARY}

Introduction: The disease of the sinuses, particularly the maxillary sinuses is common and can cause symptoms that simulate dental disease.

Objective: To determine the anatomical variations of maxillary sinus and its drainage structures involved in using computerized tomography cone beam (CTCN).

Materials and methods: The study included descriptive reviews of CTCN in 48 patients. In all cases analyzed a systematic study of the maxillary sinuses and anatomical structures involved in their drainage path, in the coronal, sagittal and axial planes.

* Profesores Facultad de Odontología, Universidad de Chile.

** Profesores Facultad de Odontología, Universidad de Antioquía. 
Results and conclusions: Morphological changes were identified in the internal maxillary sinus septum, the Haller's cell, uncinate process and ethmoid bulla. It was noted the presence of internal septum and maxillary sinus Haller cells in $76.74 \%$ and $16.28 \%$ of individuals, respectively. The horizontal uncinate process was observed in $79.07 \%$ of the subjects and the vertical at $20.93 \%$. In addition, bilateral ethmoid bullae were identified in $62.79 \%$ of the cases. The sinus anatomical variations occur mainly marked by prenatal and postnatal development, linked to the overall pneumatization neumocraneo and structures that inhibit or enable their development. Beam computed tomography cone provides images that allow visualization and proper evaluation of the maxillary sinus and osteomeatal complex.

Key words: Maxillary sinus, drainage pathway, anatomical variations, computed tomography cone-beam.

Fecha de recepción: 24 de enero de 2011.

Aceptado para publicación: 10 de febrero de 2011.

Pinares Toledo J, Carrillo Porras T, Guzmán Zuluaga IC, Ardila Medina CM, Guzmán Zuluaga CL. Variabilidad anatómica de los senos maxilares y de estructuras involucradas en su vía de drenaje. Av. Odontoestomatol 2012; 28 (2): 89-94.

\section{INTRODUCCIÓN}

Los senos paranasales son extensiones aéreas de la cavidad nasal, constituidas por los senos etmoidales, frontales, esfenoidales y maxilares (1). Los senos maxilares son las cavidades sinusales de mayor tamaño; ocupan el cuerpo del maxilar superior y se encuentran limitados por cuatro paredes, sin embargo pueden presentar tabiques internos. La pared medial del seno maxilar es la más compleja, presentando un orificio natural de drenaje (ostium maxilar) hacia el cual el moco es transportado por la actividad ciliar (2). La pared medial puede presentar además defectos descritos como ostium accesorios, los cuales corresponden a orificios que se abren en el sitio de las fontanelas nasales (2, 3). Se ha señalado que tanto el infundíbulo etmoidal como el meato medio constituyen unas de las estructuras más afectadas por variaciones anatómicas, produciendo estrechez de estas vías, facilitando a su vez procesos infecciosos (4).

La enfermedad de los senos paranasales, particularmente la de los senos maxilares, es común y puede causar sintomatología que simula patología dental. Debido a la estrecha relación entre las estructuras dentales y los senos maxilares, puede presentarse también alguna patología odontogénica que afecte los senos maxilares. De esta forma, la enfermedad sinusal es importante terapéuticamente en el campo de la medicina y la odontología (5).

Actualmente para el estudio de los senos paranasales, se utilizan algunas tecnologías avanzadas en imágenes diagnósticas que reemplazan de cierta manera a la radiología convencional (5). Es así como la tomografía computarizada ofrece una excelente delineación de la anatomía ósea de los senos paranasales. Sonkens y cols. (6), identificaron cinco patrones de enfermedad sinusal inflamatoria a través de tomografía computarizada, incluyendo un patrón infundibular que involucra enfermedades limitadas a la ruta de drenaje de los senos maxilares, con obstrucción del ostium maxilar y/o del infundíbulo etmoidal $(6,7)$. Las variantes anatómicas que pueden causar este patrón inflamatorio son las celdillas de Haller, la neumatización del proceso unciforme y la bulla etmoidal grande (6).

Por su parte, la tomografía computarizada de haz de cono (TCHC), introducida a finales del siglo XX para el estudio de las estructuras dentomaxilofaciales, permite la obtención de imágenes milimétricas a través de la adquisición de un volumen de datos que 
proporcionan información en las tres dimensiones del espacio. Debido a sus ventajas que incluyen alta resolución, baja dosis de radiación y bajo costo, comparado con la tomografía computarizada helicoidal, se ha convertido en una modalidad de diagnóstico por imagen, que permite una adecuada visión de las cavidades paranasales (8-10).

El objetivo de este estudio fue determinar la prevalencia de variaciones anatómicas del seno maxilar y de las estructuras involucradas en su drenaje, utilizando la TCHC.

\section{MATERIALES Y MÉTODOS}

El estudio incluyó los exámenes descriptivos de TCHC de 48 pacientes con edades entre 13 a 55 años. Los exámenes se realizaron en un centro de diagnóstico por imágenes de la Región Metropolitana de Santiago de Chile, entre enero de 2008 y diciembre de 2009. En el estudio se utilizó un equipo Galileos-3D Cone Beam Scanner (Sirona Dental Systems Inc., Bensheim, Alemania), con un campo de visión de $15 \mathrm{~cm}$, un volumen de reconstrucción de $15 \times 15 \times 15 \mathrm{~cm}$, matriz de $1.024 \times 1.024$ píxeles, tamaño del voxel isotrópico de $0,30 \mathrm{~mm}$, con escala de grises de 12 bit. Las imágenes fueron visualizadas mediante Microsoft Windows 7 utilizando el software Galaxis para Galileos.

En todos los casos analizados se realizó un estudio sistemático de los senos maxilares y de las estructuras anatómicas involucradas en su vía de drenaje, en los planos coronal, sagital y axial. Se evaluaron las siguientes variantes anatómicas: presencia en senos maxilares de septos internos completos e incompletos, presencia de ostium maxilares accesorios, presencia de celdillas de Haller, neumatización, orientación (vertical u horizontal) y desviación (a medial o lateral) del proceso uncinado y presencia de bulla etmoidal grande. Se consideraron como celdillas de Haller a toda aquella celdilla bajo la bulla etmoidal, a lo largo de la línea del seno maxilar y de la porción más inferior de la lámina orbitaria. Se clasificaron como grandes, aquellas bullas etmoidales que estrechaban u obstruían el infundíbulo etmoidal y el meato medio. Se excluyeron del estudio aquellos pacientes menores de 12 años (en los cuales los senos paranasales aún no alcanzan su tamaño adulto) y aquellos individuos en los cuales la anatomía de los senos maxilares y el complejo osteomeatal estaba modificada, ya sea por trauma maxilofacial, patología sinusal o por procedimientos quirúrgicos sinusales previos.

\section{RESULTADOS}

De los 48 pacientes estudiados se excluyeron los exámenes de cinco pacientes debido a imágenes borrosas del seno maxilar que impedían realizar una buena evaluación del complejo osteomeatal. De esta manera, se incluyeron en la evaluación las imágenes de 43 pacientes, 26 correspondían a mujeres $(60,47 \%)$ y 17 a hombres (39,53\%). Se identificaron variaciones morfológicas en el septo interno del seno maxilar (Figura 1), la celdilla de Haller, el proceso unciforme (Figura 2) y la bulla etmoidal.

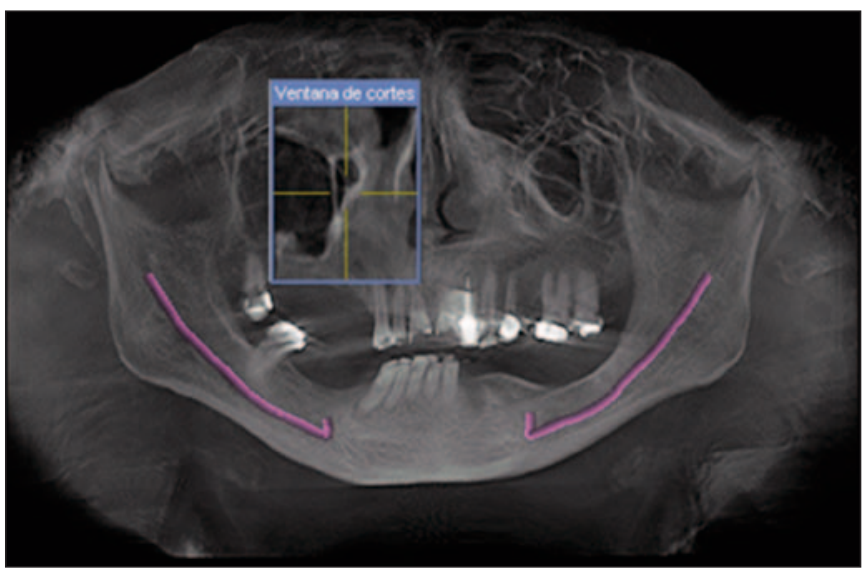

Fig. 1. Septo interno del seno maxilar.

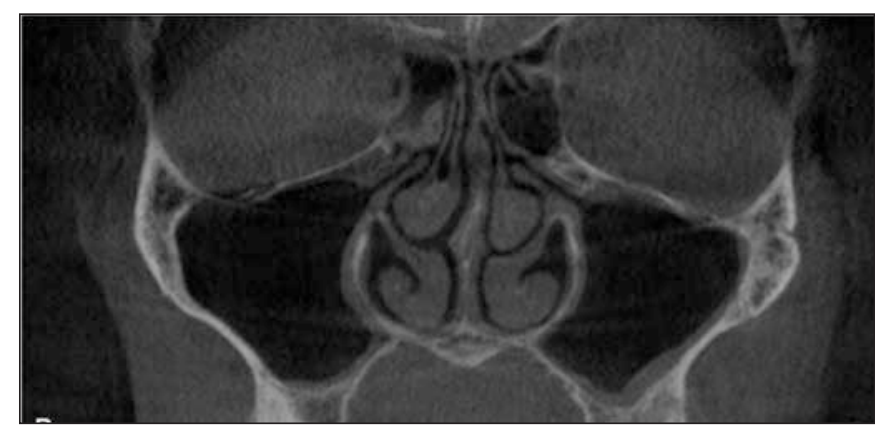

Fig. 2. Proceso unciforme horizontal con desviación hacia la línea media. 
Se observó septo interno del seno maxilar en el $76,74 \%$ de los pacientes. Se encontró septo completo bilateral en $42,86 \%$ de los sujetos y septo bilateral parcial en el 57,14\%. Se encontraron septos completos en el lado derecho $(46,67 \%)$ y en el lado izquierdo $(53,33 \%)$. La tabla 1 presenta las variaciones encontradas en el septo según el sexo. No se observó la presencia de fontanela u ostia accesoria en ningún caso.

Se identificó presencia de celdillas de Haller en el $16,28 \%$ de los sujetos. La figura 3 presenta el porcentaje de celdillas de Haller derechas, izquierdas y bilaterales según el sexo.

El proceso unciforme horizontal se observó en el $79,07 \%$ de los individuos y el vertical en el $20,93 \%$. La tabla 2 presenta el porcentaje de proceso unciforme vertical y horizontal, derecho e izquierdo según su distribución por sexo. No se observó ningún caso de proceso unciforme neumatizado. Los procesos unciformes desviados hacia la línea media se presentaron

\section{TABLA 1.- VARIACIONES ENCONTRADAS EN EL SEPTO INTERNO DEL SENO MAXILAR}

\begin{tabular}{|l|c|c|c|c|c|c|}
\hline \multirow{2}{*}{} & \multicolumn{2}{|c|}{ HOMBRES } & \multicolumn{2}{c|}{ MUJERES } & \multicolumn{2}{c|}{ TOTALES } \\
\cline { 2 - 7 } & $\mathrm{N}$ & $\%$ & $\mathrm{~N}$ & $\%$ & $\mathrm{~N}$ & $\%$ \\
\hline DERECHO COMPLETO & 7 & $9,99 \%$ & 7 & $9,09 \%$ & 14 & $18,18 \%$ \\
\hline DERECHO INCOMPLETO & 14 & $\mathbf{1 8 , 1 8 \%}$ & 17 & $22,05 \%$ & 31 & $40.26 \%$ \\
\hline ZZQUIERDO COMPLETO & 9 & $11,69 \%$ & 7 & $9,09 \%$ & 16 & $20,78 \%$ \\
\hline IZQUIERDO INCOMPLETO & 6 & $7,79 \%$ & 10 & $12,99 \%$ & 16 & $20,78 \%$ \\
\hline TOTALES & 36 & $46,75 \%$ & 41 & $53,25 \%$ & 77 & $100,00 \%$ \\
\hline BILATERAL COMPLETO & 4 & $5,19 \%$ & 2 & $2,60 \%$ & 6 & $7,79 \%$ \\
\hline BIL_ATERAL INCOMPLETO & 4 & $5,19 \%$ & 4 & $5,19 \%$ & 8 & $10.38 \%$ \\
\hline
\end{tabular}

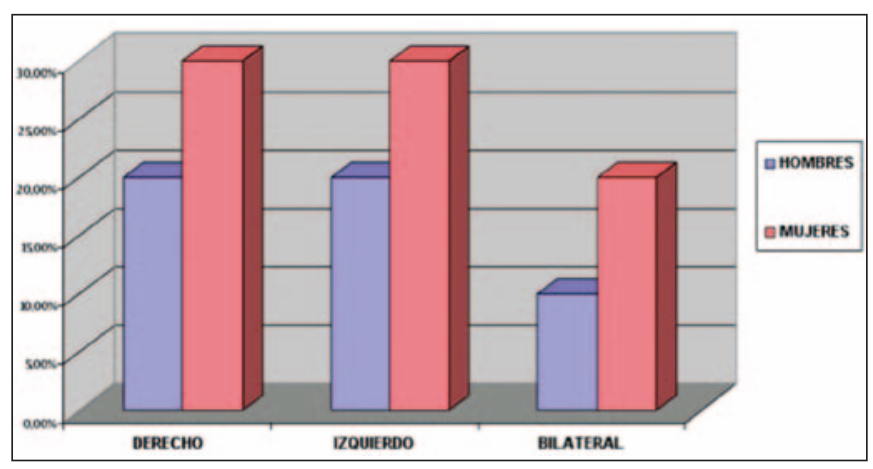

Fig. 3. Distribución de celdillas de Haller derechas, izquierdas y bilaterales según el sexo.

\section{TABLA 2.- DISTRIBUCIÓN DEL PROCESO UNCIFORME VERTICAL Y HORIZONTAL, DERECHO E IZQUIERDO SEGÚN SU DISTRIBUCIÓN POR SEXO}

\begin{tabular}{|c|c|c|c|c|c|c|}
\hline & \multicolumn{2}{|c|}{ HOMBRES } & \multicolumn{2}{|c|}{ MUUERES } & \multicolumn{2}{|c|}{ TOTALES } \\
\hline & $\mathrm{N}$ & $\%$ & Ne & $\%$ & No & $\%$ \\
\hline VERTICAL DERECHO & 3 & $1,82 \%$ & 7 & $4,24 \%$ & 10 & $6,06 \%$ \\
\hline VERTICAL IZQUIERDO & 2 & $1,21 \%$ & 6 & $3,64 \%$ & 8 & $4,85 \%$ \\
\hline HORIZONTAL DERECHO & 14 & $8,48 \%$ & 19 & $11,52 \%$ & 33 & $20,00 \%$ \\
\hline HORIZONTAL IZQUIERDO & 15 & $9,09 \%$ & 20 & $12,12 \%$ & 35 & $21,21 \%$ \\
\hline NEUMATIZADO DERECHO & 0 & $0,00 \%$ & $\mathbf{0}$ & $0,00 \%$ & 0 & $0,00 \%$ \\
\hline NEUMATIZADO IZQUIERDO & 0 & $0,00 \%$ & 0 & $0,00 \%$ & 0 & $0,00 \%$ \\
\hline DESVIACION MEDIA DERECHO & 10 & $6,06 \%$ & 17 & $10,30 \%$ & 27 & $16,36 \%$ \\
\hline DESVLACION MEDIA IZQUIERDO & 10 & $6,06 \%$ & 20 & $12,12 \%$ & 30 & $18,18 \%$ \\
\hline DESVLACION LATERAL DERECHO & 6 & $3,64 \%$ & 6 & $3,64 \%$ & 12 & $7,27 \%$ \\
\hline DESVIACION LATERAL IZQUIERDO & 6 & $3,64 \%$ & 4 & $2,42 \%$ & 10 & $6,06 \%$ \\
\hline TOTALES & 66 & $40,00 \%$ & 99 & $60,00 \%$ & 165 & $100,00 \%$ \\
\hline BIL.ATERAL VERTICAL & 2 & $1,21 \%$ & 6 & $3,64 \%$ & 8 & $4,85 \%$ \\
\hline BILATERAL HORIZONTAL. & 14 & $8,48 \%$ & 19 & $11,52 \%$ & 33 & $20,00 \%$ \\
\hline BILATERAL MEDIA & 8 & $4.85 \%$ & 17 & $10,30 \%$ & 25 & $15,15 \%$ \\
\hline BILATERAL LATERAL & 4 & $2,42 \%$ & 4 & $2,42 \%$ & 8 & $4.85 \%$ \\
\hline
\end{tabular}

en un $72,15 \%$ y los procesos unciformes desviados lateralmente en un $27.85 \%$. El proceso unciforme hipoplásico se observó en el 2,33\% de los lados.

Se identificaron bullas etmoidales bilaterales en el $62.79 \%$ de los casos. La bulla etmoidal grande bilateral se observó en un $72,73 \%$ y la bulla etmoidal grande unilateral en un $27,27 \%$. La tabla 3 presenta la distribución de bulla etmoidal según el sexo.

El proceso unciforme vertical derecho presentó bulla etmoidal grande en un $40 \%$ y el proceso unciforme izquierdo presentó bulla etmoidal grande en un $25 \%$. Una situación similar se observó en el proceso unciforme horizontal derecho, ya que la bulla etmoidal grande se encontró en el 33,33\% y $22,85 \%$, en el lado derecho e izquierdo respectivamente. La figura 4 presenta la distribución del proceso unciforme con desviación hacia la línea media derecha e izquierda,

\section{TABLA 3.- DISTRIBUCIÓN DE BULLA ETMOIDAL SEGÚN EL SEXO}

\begin{tabular}{|l|c|c|c|c|c|c||}
\hline \multirow{2}{*}{} & \multicolumn{2}{|c|}{ HOMBRES } & \multicolumn{2}{c|}{ MUJERES } & \multicolumn{2}{c||}{ TOTALES } \\
\cline { 2 - 7 } & $\mathrm{N} 0$ & $\%$ & $\mathrm{~N} \%$ & $\%$ & $\mathrm{~N} 2$ & $\%$ \\
\hline DERECHO NORMAL & 6 & $9,23 \%$ & 12 & $18,46 \%$ & 18 & $27,69 \%$ \\
\hline DERECHO GRANDE & 5 & $7,69 \%$ & 10 & $15,38 \%$ & 15 & $23,07 \%$ \\
\hline IZQUIERDO NORMAL & 9 & $13,85 \%$ & 13 & $20,00 \%$ & 22 & $33,85 \%$ \\
\hline IZQUIERDO GRANDE & 4 & $6,15 \%$ & 6 & $9,23 \%$ & 10 & $15,38 \%$ \\
\hline TOTALES & 24 & $36,92 \%$ & 41 & 63,08 & 65 & $100,00 \%$ \\
\hline BIL_ATERAL NORMAL & 4 & $6,15 \%$ & 9 & $13,85 \%$ & 13 & $20,00 \%$ \\
\hline BILATERAL GRANDE & 2 & $3,08 \%$ & 6 & $9,23 \%$ & 8 & $12,31 \%$ \\
\hline
\end{tabular}




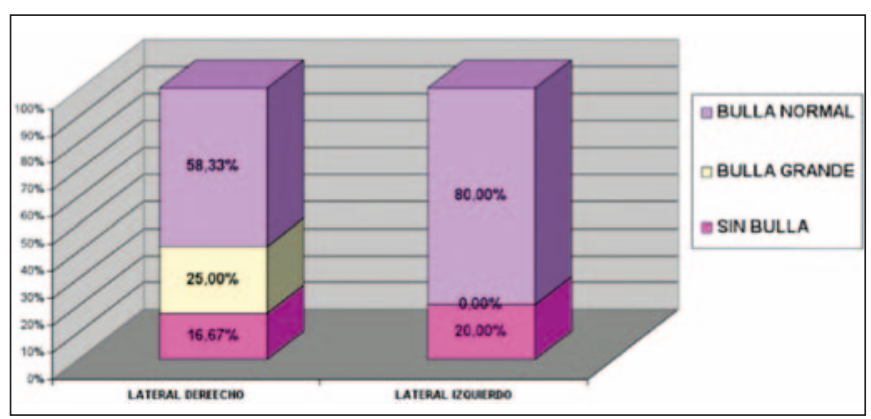

Fig. 4. Distribución del proceso unciforme con desviación hacia la línea media derecha e izquierda, según presencia de bulla del mismo lado.

según presencia de bulla del mismo lado. El proceso unciforme con desviación hacia la línea media derecha presentó una bulla etmoidal grande en un $42,31 \%$. Hacia la izquierda la bulla etmoidal grande se presentó en un $30 \%$.

La presencia de bulla etmoidal y de celdilla de Haller ocurrió en el $15,15 \%$ y $15,63 \%$ de los casos, en el lado derecho e izquierdo respectivamente.

\section{DISCUSIÓN}

La presente investigación determinó la prevalencia de variaciones anatómicas del seno maxilar y de las estructuras involucradas en su drenaje.

En este estudio se observó septo interno del seno maxilar en el $76.74 \%$ de los pacientes evaluados. Una revisión sistemática reciente identificó una prevalencia de septo entre el $21.6 \%$ y $66.7 \%$ de los casos (11). La mayoría de estudios incluidos en esa revisión, usaron radiografías panorámicas, lo cual corrobora lo descrito por algunos autores que indican la baja confiabilidad de ese tipo de radiografías, en la detección de septo en el interior del seno maxilar (11). Underwood (12) notó que la localización del septo interno del seno maxilar se presenta debido a su desarrollo en tres diferentes momentos de la erupción dental. Shibli y cols. (13), corroboran lo observado en el presente estudio, indicando que la prevalencia de septo no tiene relación con la edad ni el sexo del sujeto.

En el presente estudio se identificó presencia de celdillas de Haller en el 16,28\% de los sujetos, preva- lencia similar a la encontrada por otros investigadores (14). Es importante tener presente que el desarrollo de las celdillas de Haller puede bloquear la entrada al seno maxilar o lateralizar la pared medial del mismo.

Zhu y cols. (15), observaron prevalencias de procesos unciformes similares a las encontradas en el presente estudio. El proceso unciforme se encuentra neumatizado en un 2,5\% de los casos (15), y de acuerdo a la medida de esta neumatización, puede llegar a obstruir el drenaje de los senos maxilares.

En la presente investigación se encontraron frecuencias de bullas etmoidales similares a las descritas por otros investigadores $(16,17)$. Se encuentra neumatizada en un 65-70\%, con gran variación en tamaño y extensión. En algunos casos se han encontrado dehiscencias en la pared lateral de la bulla, sobre la lámina papirácea, con herniación de la grasa orbitaria $(16,17)$.

Los senos paranasales presentan variaciones anatómicas marcadas fundamentalmente por su desarrollo prenatal y postnatal, ligados a la neumatización general del neumocráneo y a estructuras que inhiben o permiten su desarrollo. El conocimiento previo a una cirugía, a través de estudios radiológicos, es de importancia relevante para el éxito del acto quirúrgico. El empleo de tomografías computarizadas de haz de cono como herramienta para realizar estudios morfológicos y morfométricos del seno maxilar se debe principalmente a las ventajas del tipo de imagen. La observación de una región extensa del seno maxilar, incluyendo áreas y estructuras anatómicas adyacentes, permiten un análisis preciso de su morfología y sus relaciones con puntos anatómicos de referencia. La superioridad de calidad de imagen de la TCHC es indiscutible, debido a que posibilita la diferenciación entre las estructuras dentarias y genera menos artefactos de estructuras metálicas.

\section{BIBLIOGRAFÍA}

1. Sato I, Kawai T, Yoshida S, Miwa Y, Imura K, Asaumi R, et al. Observing the bony canal structure of the human maxillary sinus in 
Japanese cadavers using cone beam CT. Okajimas Folia Anat Jpn 2010;87:123-8.

2. Al-Swiahb JN, Al Dousary SH. Computer-aided endoscopic sinus surgery: a retrospective comparative study. Ann Saudi Med 2010;30: 149-52.

3. Earwaker J. Anatomic variants in sinonasal CT. RadioGraphics 1993;13:381-415.

4. Zinreich SJ, Kennedy DW, Rosenbaum AE, Gayler BW, Kumar AJ, Stammberger H. Paranasal sinuses: CT imaging requirements for endoscopic surgery. Radiology 1987;163:769-75.

5. Granström G, Gröndahl HG. Imaging of osseointegrated implants in the temporal bone by accuitomo 3-dimensional cone beam computed tomography. Otol Neurotol 2011;32: 199-203.

6. Sonkens JW, Harnsberger HR, Blanch GM, Babbel RW, Hunt S. The impact of screening sinus CT on the planning of functional endoscopic sinus surgery. Otolaryngol Head Neck Surg 1991;105:802-13.

7. Vogl TJ, Harth M, Siebenhandl P. Different imaging techniques in the head and neck: Assets and drawbacks. World J Radiol 2010;2:224-9.

8. Quereshy FA, Savell TA, Palomo JM. Applications of Cone Beam Computed Tomography in the Practice of Oral and Maxillofacial Surgery. J Oral Maxillofac Surg 2008; 66:791-6.

9. De Vos W,Casselman J, Swennen RJ. Cone-beam computerized tomography (CBCT) imaging of the oral and maxillofacial region: A systematic review of the literature. Int. J. Oral Maxillofac Surg 2009; 38: 609-25.

10. Scarfe WC, Farman AG, Sukovic P. Clinical aplications of Cone-Beam Computed Tomography in dental practice. J Can Dent Assoc 2006;72:7580 .

11. Maestre-Ferrín L, Galán-Gil S, Rubio-Serrano M, Peñarrocha-Diago $M$, Peñarrocha-Oltra D. Maxillary sinus septa: a systematic review. Med Oral Patol Oral Cir Bucal 2010 1;15:e383-6.

12. Underwood AS. An Inquiry into the Anatomy and Pathology of the Maxillary Sinus. J Anat Physiol 1910;44:354-69.

13. Shibli JA, Faveri M, Ferrari DS, Melo L, Garcia RV, D'Avila S, et al. Prevalence of maxillary sinus septa in 1024 subjects with edentulous upper jaws: a retrospective study. J Oral Implantol 2007;33: 293-6.

14. Rysz M, Bakoñ L. Maxillary sinus anatomy variation and nasal cavity width: structural computed tomography imaging. Folia Morphol (Warsz) 2009;68:260-4.

15. Zhu SH, He GX, Sun H, Ma YH, Liu GH, Jiang M. Causes and revision endoscopic sinus surgery for recurrent sinusitis. Zhong Nan Da Xue Xue Bao Yi Xue Ban 2006;31(2):208-11.

16. Sirikçi A, Bayazit YA, Bayram M, Kanlikama M. Ethmomaxillary sinus: a particular anatomic variation of the paranasal sinuses. Eur Radiol 2004; 14:281-5.

17. Liu X, Zhang G, Xu G. Anatomic variations of the ostiomeatal complex and their correlation with chronic sinusitis: CT evaluation. Zhonghua $\mathrm{Er} \mathrm{Bi}$ Yan Hou Ke Za Zhi. 1999;34:143-6.

\section{CORRESPONDENCIA}

Carlos M. Ardila M

Calle 64, No 52-59

Medellín, Colombia.

e-mail: martinardila@gmail.com 